\title{
Validez del Doppler transcraneal en el diagnóstico de muerte encefálica
}

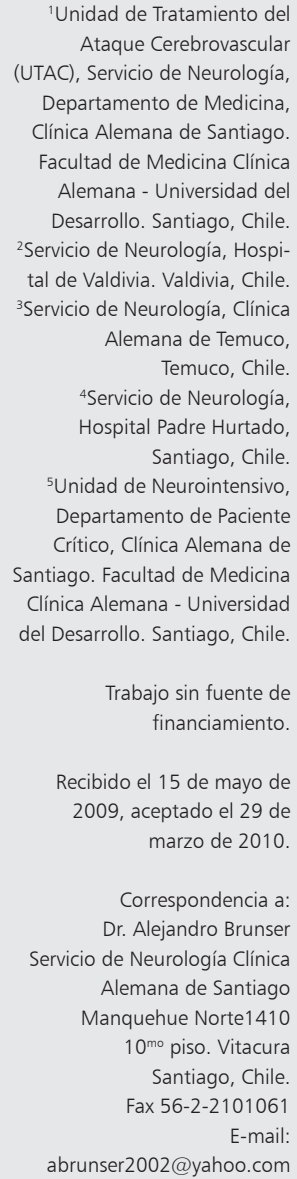

\author{
ALEJANDRO BRUNSER ${ }^{1}$, ARNOLD HOPPE ${ }^{1}$, DANIEL A. CÁRCAMO ${ }^{1}$, \\ PABLO M. LAVADOS ${ }^{1}$, ANDRÉS ROLDÁN ${ }^{2}$, RODRIGO RIVAS ${ }^{3}$, \\ MARCELA VALENZUELA ${ }^{4}$, JOSÉ MIGUEL MONTES ${ }^{5}$
}

\section{Validation of transcranial Doppler in the diagnosis of brain death}

Background: The clinical diagnosis of brain death is complex. Aim: To evaluate the diagnostic accuracy of transcranial Doppler (TCD) for brain death. Patients and Methods: Patients seen on the intensive care unit of a private hospital between January 2004 to December 2008, were included if they were in structural coma, had no craniectomy and had a blind evaluation by a neurologist and TCD done in less than three hours. The diagnosis of brain death was based on a clinical evaluation that considered the absence of sedative drugs, median blood pressure $>60 \mathrm{mmHg}$, body temperature over $35^{\circ}$ Celsius and complete absence of brainstem reflexes. An expert neurosonologist, with a TCD-PMD-100, $2 \mathrm{Mhz}$ transducer, used an institutional protocol that considers the examination as positive for brain circulatory arrest when there is presence of reverberating, small systolic peaks or the disappearance of a previous signal present on both middle cerebral arteries and intracranial vertebral arteries. Results: Fifty three patients were evaluated, 25 with clinical brain death. On 45 cases (84.9\%), the interval between both evaluations was less than one hour. The sensitivity, specificity, positive and negative predictive values for the diagnosis of brain death with TCD were 100, 96, 96.1 and 100\% respectively. Positive and negative likelihood ratios for brain death were 28 and 0, respectively. Conclusions: TCD is a valid and useful technique for the diagnosis of brain death and can be used on complicated cases.

(Rev Med Chile 2010; 138: 406-412).

Key words: Brain, death; Intensive care units; Transcranial Doppler.
$\mathrm{L}$ a determinación de la muerte encefálica (ME) en Chile, así como en la mayoría de los países del mundo, es clínica ${ }^{1,2}$. Las exigencias clínicas que permiten al médico documentar la abolición irreversible de todas las funciones encefálicas comprenden la constatación clínica del coma, la ausencia de reflejos troncoencefálicos y la apnea. Las circunstancias que podrían restar confiabilidad al examen clínico se encuentran bien tipificadas: intoxicaciones por sedantes, trastornos metabólicos severos, hipotermia importante y el uso de bloqueadores musculares. Existen otras condiciones en las que la exploración neurológica integral y certera resulta imposible. Es el caso de traumatismos faciales u oculares severos que impiden explorar los reflejos troncoencefálicos por lesión directa de los efectores o traumas tóracopulmonares graves que imposibilitan realizar el test de apnea en forma segura ${ }^{3-5}$. En todas estas circunstancias el médico se ve obligado a recurrir a métodos de apoyo tecnológicos para establecer el diagnóstico de ME. La angiografía convencional radiológica y la angiografía isotópica son técnicas que permiten documentar la ausencia de flujo intracraneal lo que constituye condición suficiente para el diagnóstico de $\mathrm{ME}^{6,7}$. Sin embargo, ambas 
requieren trasladar al paciente fuera de la Unidad de Tratamiento Intensivo y tienen un alto costo, a lo que se agrega el inconveniente, en el caso de la angiografía convencional, de ser invasiva. Otras técnicas como la angiografía por tomografía axial computada aun no han sido suficientemente validadas ${ }^{8,9}$.

El Doppler transcraneano (DTC) es una técnica de exploración de los vasos intracraneales, no invasiva, repetible, de bajo costo y que se puede realizar al borde de la cama de los pacientes. Ha demostrado ser útil en el diagnóstico de oclusiones vasculares cerebrales agudas, hipertensión intracraneana y en el diagnóstico y seguimiento del vasoespasmo por hemorragia subaracnoídea, entre otras patologías ${ }^{10-12}$.

En diversas publicaciones se ha establecido la alta sensibilidad y especificidad del DTC en el diagnóstico del paro circulatorio cerebral comparado con angiografía ${ }^{13-15}$. No obstante, su correlación con el diagnóstico clínico de ME, genuino gold standard de la condición, ha sido escasamente estudiado ${ }^{16}$. Nuestro objetivo fue investigar la validez del DTC en la determinación del paro circulatorio intracraneal y su relación con el establecimiento de ME por criterios neurológicos en pacientes sometidos a evaluación por coma de causa estructural.

\section{Pacientes y Métodos}

En la Unidad de Cuidados Intensivos de Clínica Alemana (UCI) el procedimiento protocolizado ante un paciente en coma profundo por una lesión encefálica estructural documentada, en quien se sospecha ME, es la solicitud de una evaluación clínica por parte del neurólogo de turno y la realización de un DTC. Por disposición del laboratorio de Neurosonología, a partir de enero de 2004 ambas evaluaciones se realizan sistemáticamente por evaluadores distintos y en forma ciega, para evitar influencias mutuas en pacientes que podrían ser potenciales donantes de órganos. Se procura siempre realizar ambas evaluaciones con el menor intervalo de tiempo posible. Los resultados de estas evaluaciones son registrados prospectivamente. Tanto la evaluación clínica, como el DTC fueron ciegos e independientes de los resultados del otro evaluador. Los pacientes fueron incluidos de manera prospectiva consecutiva desde enero 2004 hasta diciembre 2008. Ambas evaluaciones fueron realizadas lo antes posible.
Los criterios de inclusión para el presente análisis fueron: 1) paciente hospitalizado en la UCI; 2) coma de causa estructural documentada, con sospecha de muerte encefálica; 3) ausencia de craniectomía descompresiva; 4) posibilidad de acceder a las ventanas sonográficas temporales y suboccipital para evaluación de la circulación carotídea y vértebro-basilar; 5) evaluación clínica factible de realizar en forma completa, incluyendo todos los reflejos troncoencefálicos y el test de apnea; 6) intervalo entre la evaluación clínica y sonográfica menor a 3 horas.

El diagnóstico de ME se realizó de acuerdo con las normativas establecidas en el reglamento Decreto Supremo No 656 de la Ley 19.451. Se constató una temperatura corporal sobre $35^{\circ} \mathrm{C}$, se descartó la presencia de alteraciones metabólicas severas, de intoxicación por fármacos sedantes y uso de bloqueadores musculares. Se estableció la presencia de coma por la ausencia de respuesta al estímulo nociceptivo profundo. Se documentó la ausencia de reflejos fotomotores, corneales, oculocefálicos, oculovestibulares y traqueal. Se realizó el test de apnea considerando concordante con el diagnóstico de ME la ausencia de esfuerzo respiratorio con una $\mathrm{PaCO}_{2}$ de $60 \mathrm{mmHg}$ documentada con gasometría arterial.

El DTC fue realizado por un único neurosonólogo (AB) utilizando para esto un equipo de DTC del tipo power motion PMD-100 Spencer technologies, con un transductor pulsado de $2 \mathrm{MHz}$, a una potencia de $100 \%$ y con una amplitud de muestreo de 6 milímetros y un filtro en 200, que en caso de no encontrarse flujo diastólico podía ser reducido a 25. Se utilizó un protocolo estandarizado ${ }^{17}$ que analiza las arterias cerebrales medias en su segmento M1 medio (profundidad entre 53 y 55 $\mathrm{mm}$ ) a través de la ventana temporal explorando 1 centímetro por delante y medio centímetro por arriba del trago con una dirección del transductor ligeramente hacia arriba y anterior. Las arterias vertebrales intracraneanas fueron exploradas por ventana suboccipital a $60 \mathrm{~mm}$ de profundidad, localizando el transductor 1 centímetro medial a la apófisis mastoides apuntando hacia la raíz de la nariz. La Figura 1 demuestra las diferentes ventanas de exploración en el DTC. El examen fue concordante con paro circulatorio cerebral si en todas las arterias evaluadas el patrón de señal fue: 1) Flujo oscilante: Señal de flujo diastólico reverso (reverberante);2) Espiga sistólica de pequeña mag- 


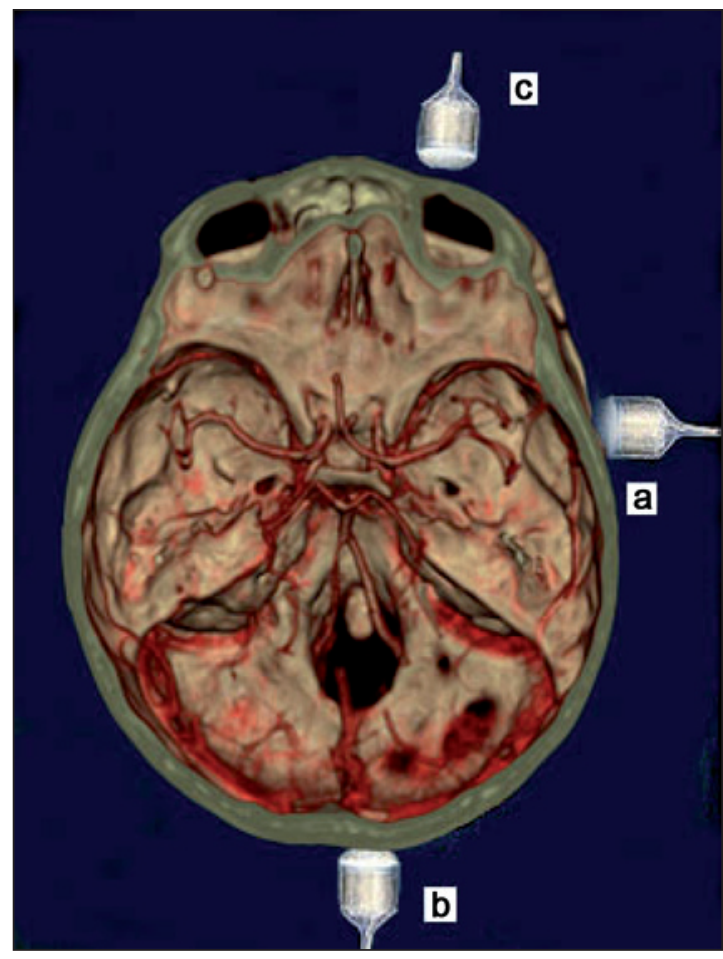

Figura 1. Ventanas habituales usadas en la realización de un examen de Doppler transcraneano: a) Ventana temporal; b) Suboccipital; c) Ventana transorbitaria (utilizada frecuentemente en estudio de enfermedad cerebro-vascular).

nitud: Señal de flujo anterógrado sistólica pequeña, con ausencia de flujo diastólico; 3) Ausencia de señal: La ausencia de señal sólo se consideró como evidencia de ausencia de flujo en arterias en que existió documentación de flujo en un estudio de DTC previo.

Si bien no existió límite de tiempo en la realización del examen, en el caso de los comas causados por hipertensión intracraneana aguda, el examen fue extendido siempre por más de 30 minutos realizando una nueva evaluación de todas las arterias evaluadas luego de ese tiempo.

En el análisis incluimos todos los pacientes evaluados de manera consecutiva entre enero 2004 y diciembre de 2008.

\section{Análisis}

El resultado de la exploración clínica fue definida como gold standard y dicotomizado en pacientes "con muerte encefálica" (ME) y "no muerte encefálica" (No-ME). Se calculó la sensi-
Tabla 1. Características demográficas y causas de fallecimiento de la muestra de pacientes con muerte encefálica

\begin{tabular}{|lc|}
\hline Variables & $\mathbf{n}=\mathbf{2 5}$ \\
\hline Edad promedio y rango (años) & $49(18-89)$ \\
Hombre/mujeres ( $\mathrm{n})$ & $14 / 11$ \\
\hline Causa de fallecimiento & 7 \\
Hemorragia intracraneana & 6 \\
$\begin{array}{l}\text { Traumatismo encéfalo-craneano } \\
\text { grave }\end{array}$ & 6 \\
$\begin{array}{l}\text { Hemorragia subaracnoídea aneu- } \\
\text { rismática }\end{array}$ & 3 \\
$\begin{array}{l}\text { Ataque cerebrovascular isquémico } \\
\text { Encefalopatía hipóxica isquémica }\end{array}$ & 2 \\
$\begin{array}{l}\text { Tumor intracraneano } \\
\text { Tiempo en minutos entre examen } \\
\text { clínico y DTC }\end{array}$ & 1 \\
\hline
\end{tabular}

bilidad, especificidad, valor predictivo positivo y negativo del DTC, para la determinación de la ME. Se calculó el cociente de probabilidad (likelihood ratio $)^{18}$ positiva y negativa para la determinación de ME por el DTC.

\section{Resultados}

Durante el período de estudio fueron evaluados 86 pacientes, de los cuales 19 fueron excluidos por presentar un intervalo entre ambas evaluaciones superior a 3 horas, 5 pacientes por presentar craniectomías o drenajes craneanos, 2 pacientes por no tener estudio de DTC, 2 pacientes por no poder realizar el test de apnea, 2 pacientes por uso de relajantes musculares que impidieron el examen clínico y finalmente 2 pacientes fueron excluidos por no encontrase en el DTC inicial señal en las arterias cerebrales medias bilaterales, no pudiendo descartarse que correspondieran a ventanas sonográficas no óptimas. De los 53 pacientes restantes, 28 pacientes correspondieron a No-ME en coma, 25 fueron diagnosticados con $\mathrm{ME}$. El examen clínico fue realizado en 37 casos $(69,8 \%)$ antes que el estudio de DTC. La diferencia de tiempo entre ambos fue de 45,9 minutos DS $\pm 55,9$ (5-179). En 45 casos (84,9\%) el intervalo entre ambas evaluaciones fue inferior a una hora. 


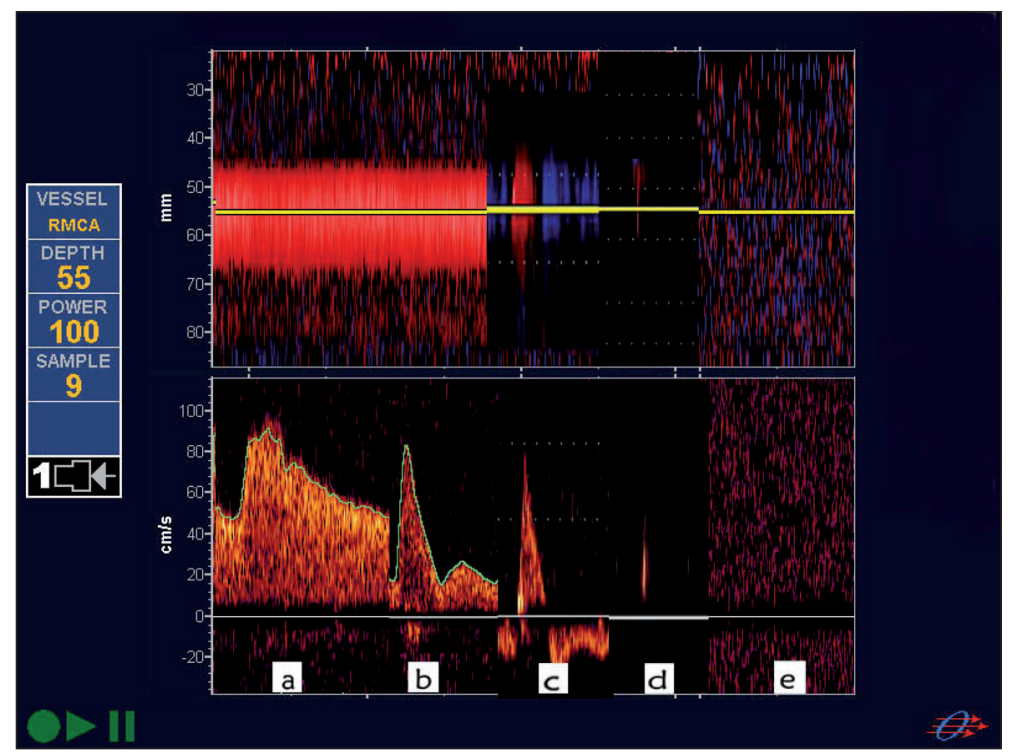

Figura 2. Progresión de hipertensión intracraneana por DTC: a) Corresponde a curvas de tipo normal; b) Curva de tipo hipertensiva intracraneana con disminución de la velocidad de diástole, la sístole adquiere un aspecto puntiagudo; c) Curva de tipo reverberante con flujo positivo en sístole y negativo en diástole; d) Curva de pequeños peaks sistólicos, hay una pequeña señal sistólica en sístole; e) Ausencia total de flujo tanto en sístole como en diástole.
Las características de la población con $\mathrm{ME}$ se muestra en la Tabla 1. El DTC demostró paro circulatorio intracraneal en 26 pacientes, de los cuáles 25 correspondieron a pacientes con ME en la evaluación concomitante. Existió un caso de falso positivo al DTC.

El DTC fue concordante con el diagnóstico de ME con una sensibilidad de 100\% (IC 95\% 83,4-100) y una especificidad de $96 \%$ (IC 95\% $79,8-99,8)$. El valor predictivo positivo fue 96,1 (IC 95\% 78,4-99,8) y el negativo 100 (IC 95\% 84,5-100). El cociente de probabilidad positivo fue 28 (IC 95\% 4,0-90,0) y el cociente de probabilidad negativo fue $0(0,0-0,32)$. El patrón sonográfico más frecuentemente encontrado en los pacientes con ME fue el de "flujo reverberante" en 70 de100 arterias evaluadas. En 21 arterias se encontró un patrón de "peaks sistólicos pequeños" y en las restantes 9 arterias se encontró "ausencia de flujo en una arteria con flujo previo documentado".

La Figura 2 muestra el deterioro del flujo cerebral al DTC.

\section{Discusión}

Este estudio demuestra que el DTC posee una muy alta sensibilidad y especificidad comparado con el diagnóstico clínico de ME en pacientes en coma por lesiones estructurales. Los valores del cociente de probabilidad positiva de 28 y negativa de 0 , demuestran que el DTC es una técnica con una alta capacidad para distinguir sujetos con ME dentro de una serie de pacientes con coma estructural.

Nuestros resultados son concordantes con otras series comunicadas, como lo demuestra la Tabla 2. En un reciente metaanálisis Monteiro y cols ${ }^{16}$ identificaron 233 artículos publicados que de una u otra forma abordan la validez del DTC para el diagnóstico de muerte encefálica. Para valorar la calidad de los diferentes estudios los autores aplican los siguientes criterios: a) presencia de una comparación independiente y ciega con un patrón oro de referencia; b) la población estudiada debía incluir un espectro de pacientes semejante a aquellos a quienes se podría aplicar el test en la práctica clínica; c) inclusión de pacientes consecutivos que cumplieran los criterios de inclusión; y d) descripción suficiente de la metodología DTC utilizada como para permitir la reproducción del método. Los estudios que cumplían los cuatro criterios fueron tipificados como de alta calidad; si cumplían los criterios a y d se los consideró de menor calidad, mientras que el resto de los estudios fueron considerados no válidos. Con este tamiz sólo 10 estudios se consideraron válidos para determinar la utilidad del DTC y sólo 2 fueron ca- 
Validez del Doppler transcraneal en el diagnóstico de muerte encefálica - A. Brunser et al

Tabla 2. Estudios que evalúan Doppler Transcraneano en muerte encefálica

\begin{tabular}{|lcccccl|}
\hline Autor & $\begin{array}{c}\text { n de } \\
\text { pacientes }\end{array}$ & Sn & Es & VPP & VPN & Circulación examinada \\
\hline Zurynski $^{13}$ & 133 & 93,2 & 100 & 100 & 80,56 & Anterior y posterior \\
Paolini' $^{14}$ & 15 & 100 & 100 & 100 & 100 & Sólo arteria cerebral media \\
\hline Feri' $^{19}$ & 37 & 81,8 & 100 & 100 & 78,9 & Sólo arteria cerebral media \\
Domínguez-Roldán $^{20}$ & 75 & 84,6 & 100 & 100 & 92,4 & Sólo arteria cerebral media \\
Hadani $^{21}$ & 137 & 96,3 & 98,1 & 98,7 & 94,6 & Anterior y posterior \\
Petty $^{22}$ & 54 & 80 & 100 & 100 & 84 & Anterior y posterior \\
\hline Brunser & 53 & 100 & 96 & 96.1 & 100 & Anterior y posterior \\
\hline
\end{tabular}

Sn: sensibilidad, Es: especificidad, VPP: valor predictivo positivo, VPN: valor predictivo negativo.

lificados como de alta calidad. En estos 2 estudios la sensibilidad fue de 93,2 a $95 \%$ y la especificidad de 98,1 a 100\% (Hadani 1999, Zurynsiki 1991).

Nuestra serie cumple con los criterios de calidad definidos por Monteiro y alcanza una sensibilidad mayor que los 2 estudios previos. Uno de los factores que pueden haber contribuido a esta mayor sensibilidad es el avance tecnológico que significa la incorporación del modo $\mathrm{M}^{23}$, que facilita notablemente la identificación de las arterias intracraneales, su exploración longitudinal y la determinación de flujos arteriales intracerebrales mínimos. La alta especificidad se fundamenta en el requisito de incluir la exploración de la circulación anterior y posterior. Este criterio es más estricto que el utilizado en otros estudios en que se exige la evaluación de al menos 2 arterias pero que pueden ser ambas de la circulación anterior ${ }^{19,20}$, lo anterior es respaldado por el hecho de la presencia de un solo falso positivo en 53 casos evaluados. Este caso correspondió a un paciente que presentó un paro circulatorio intracraneal al DTC con un examen clínico que no cumplía aún con todos los criterios clínicos de ME al documentarse la presencia de esfuerzos respiratorios en el test de apnea. Este paciente presentaba una extensa hemorragia cerebelosa con compresión de tronco de 6 horas de evolución al momento de la evaluación clínica y sonográfica. El paciente perdió esta reactividad en el lapso de 24 horas después del DTC, completando los criterios de ME.

La ausencia de flujo sólo fue aceptada como evidencia de paro circulatorio si en las mismas arterias se había demostrado en un estudio previo la presencia de flujo. Esto permite discriminar la ausencia de ventana acústica, hecho encontrado en nuestra población chilena en una muestra de 400 personas en $8,7 \%$ para la circulación anterior y $12,3 \%$ para la circulación posterior por medio de la ventana suboccipital (Brunser. World Congress of Stroke Cape Town, South Africa, October 2006), cifras muy concordantes con estudios internacionales ${ }^{24,25}$.

En la presente serie el intervalo entre la realización del DTC y el examen clínico fue menor a 3 horas, lo cual subraya la utilidad del DTC como un estudio que no requiere de latencia para su realización y validez.

Los estudios complementarios para el diagnóstico de ME pueden clasificarse entre aquellos que documentan paro circulatorio cerebral (angiografía convencional e isotópica) y aquellos que documentan pérdida de función eléctrica encefálica (EEG, potenciales evocados). El electroencéfalograma (EEG) en particular tiene el inconveniente de que varias condiciones de las que suelen impedir la utilización de la mera evaluación clínica, como la presencia de hipotermia, intoxicación por depresores del sistema nervioso central y trastornos metabólicos graves, también alteran el EEG por lo que invalidan su uso ${ }^{26}$. Entre los estudios que documentan paro circulatorio cerebral la reglamentación chilena contenida en el decreto del Ministerio de Salud considera hasta la fecha sólo la angiografía convencional y la angiografía isotópica. Ambas técnicas tienen el inconveniente de que requieren trasladar al paciente fuera de la unidad de intensivo, lo que implica alto riesgo y 
también elevados costos. En contraste con ello el DTC es una técnica de bajo costo, no invasiva y que se puede realizar al lado de la cama del paciente crítico. Dada su alta sensibilidad y especificidad esta técnica podría ser usada como complemento del examen clínico en pacientes en los que pudiera no ser evaluable algún reflejo de tronco, por ejemplo por lesiones faciales que impidan el examen ocular o por tener traumas toráxicos complicados que impidan la realización del test de apnea. Creemos firmemente la incorporación de esta técnica en la normativa nacional está justificada plenamente. Sin embargo, existen algunas desventajas como la ausencia de DTC en la mayoría de los hospitales de nuestro país, la falta de operadores calificados y el hecho de ser operador dependiente.

Nuestro estudio posee fortalezas como haber sido realizado en una instancia clínica habitual, con un número importante de enfermos y con mínima diferencia de tiempo entre la evaluación clínica y ultrasonográfica. Su gran limitación es la presencia de un solo operador de DTC lo que resta reproducibilidad a estos resultados, y en segundo lugar un tamaño de muestra limitado.

\section{Referencias}

1. Anonymous. Diagnosis of brain death. Statement issued by the honorary secretary of the conference of Royal Medical Colleges and their Faculties in the United Kingdom on 11 October 1976. BMJ 1976; 2: 1187.

2. Anonymous. Richtlinien zur Feststellung des Hirntodes. Dritte Fortschreibung 1997 mit Ergänzungen gemä $\beta$ Transplantationgesetz (TPG). Wissenschaftlicher Beirat der Bundesärztekammer. Dtsch Ärztebl 1998; 95: B-1509.

3. Anonymous. Guidelines for the determination of brain death in children. Task Force for the Determination of Brain Death in Children. Neurology 1987; 37: 1077.

4. Wijdicks E. Clinical diagnosis and confirmatory testing of brain death in adults In: Brain Death: a clinical guide. Ed Eelco Wijdicks. Lippincott Williams \& Wilkins 2001; p 61-90.

5. Hoppe A. Muerte encefálica. Tratado de Neurología Clínica. Eds Nogales-Gaete J, Donoso A, Verdugo R. Sociedad de Neurología, Psiquiatría y Neurocirugía. Editorial Universitaria S.A. 2005; p 57-65.

6. Bradac GB, Simon RS. Angiography in brain death. Neuroradiology 1974; 7: 25-8.

7. Kricheff II, Braunstein P, Korein J, George AE, Kumar
AJ. Isotopic and angiographic determination of cerebral blood flow. A correlation in patients with cerebral death. Acta Radiol Suppl 1976; 347: 119-29.

8. Qureshi AI, Kirmani JF, Xavier AR, Siddiqui AM. Computed tomographic angiography for diagnosis of brain death. Neurology 2004; 62: 652-3.

9. Quesnel C, Fulgencio JP, Adrie C, Marro B, Payen L, Lembert N, et al. Limitations of computed tomographic angiography in the diagnosis of brain death. Intensive Care Med 2007; 33: 2129-35.

10. Demchuk AM, Christou I, Wein TH, Felberg RA, Malkoff M, Grotta JC, et al. Accuracy and criteria for localizing arterial occlusion with transcranial Doppler. J Neuroimaging 2000; 10: 1-12.

11. Lysakowski C, Walder B, Costanza M, Tramer M, Phil D. Transcranial Doppler Versus Angiography in Patients With Vasospasm due to a Ruptured Cerebral Aneurysm: A Systematic Review. Stroke 2001: 32; 2292-8.

12. Shigemori M, Kikuchi N, Tokutomi, T, Ochiai S, Harada $\mathrm{K}$, Kikuchi T, et al. Monitoring of severe head-injured patients with transcranial Doppler (TCD) ultrasonography. Acta Neurochir Suppl (Wien) 1992; 55: 6-7.

13. Zurynski Y, Dorsch N, Pearson I, Choong R. Transcranial Doppler ultrasound in brain death: experience in 140 patients. Neurol Res 1991; 13: 248-52.

14. Paolin A, Manuali A, Di Paola F, Boccaletto F, Caputo P, Zanata R, et al. Reliability in diagnosis of brain death. Intensive Care Med 1995; 21: 657-62.

15. Van Velthoven V, Calliau L. Diagnosis of brain death. Transcranial Doppler sonography as an additional method. Acta Neurochir (Wein) 1998; 95: 57-60.

16. Monteiro LM, Bollen CW, van Huffelen AC, Ackerstaff RG, Jansen NJ, van Vught AJ. Transcranial Doppler ultrasonography to confirm brain death: a meta-analysis. Intensive Care Med 2006; 32: 1937-44.

17. Garami Z, Calleja S, Malkoff M, Alexandrov A, Improved Transcranial Doppler (TCD) protocol for diagnosis of Cerebral Circulatory Arrest in Brain Death Patients. J Neuroimaging 2003; 13: 178.

18. Deeks, Jonathan J. Senior research biostatistician 1; Altman, Douglas G professor of statistics in medicine 2 Diagnostic tests 4: likelihood ratios. BMJ 2004; 329 : 168-9.

19. Feri M, Ralli L, Felici M, Vanni D, Capria V. Transcranial Doppler and brain death diagnosis. Crit Care Med 1994; 22: $1120-6$.

20. Domínguez-Roldán JM, Murillo-Cabezas F, MuñozSánchez A, Santamaría-Mifsut JL, Villen-Nieto J. Changes in the Doppler waveform of intracranial arteries in patients with brain-death status. Transplant Proc 1995; 27: 2391-2. 
21. Hadani M, Bruk B, Ram Z, Knoller N, Spiegelmann R, Segal E. Application of transcranial Doppler ultrasonography for the diagnosis of brain death. Intensive Care Med 1999; 25: 822-8.

22. Petty GW, Mohr JP, Pedley TA, Tatemichi TK, Lennihan L, Duterte DI, et al. The role of transcranial Doppler in confirming brain death: sensitivity, specificity, and suggestions for performance and interpretation. Neurology 1990; 40: 300-3.

23. Moehring MA, Spencer MP. Power M-mode Doppler (PMD) for observing cerebral blood flow and tracking emboli. Ultrasound Med Biol 2002; 28: 49-57.
24. Itoh T, Matsumoto M, Handa N, et al. Rate of successful recording of blood flow signals in the middle cerebral artery using transcranial Doppler sonography. Stroke 1993; 24: 1192-5.

25. Marinoni M, Ginanneschi A, Forleo P, Amaducci L. Technical limits in transcranial Doppler recording: inadequate acoustic windows. Ultrasound Med Biol 1997; 23: $1275-7$.

26. Segura T, Jiménez P, Jerez P, García F, Córcoles V. Prolonged clinical pattern of brain death in patients under barbiturate sedation: usefulness of transcranial Doppler. Neurología 2002; 17: 219-22. 